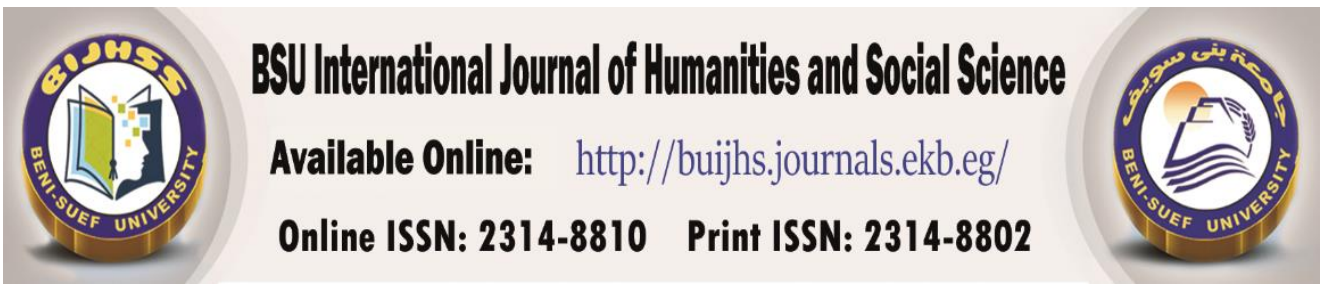

\title{
La figure du héros: étude comparée entre " Et les chiens se taisaient" d'Aimé Césaire et " La tragédie d'Al-Hallaj" de Salah Abdel Sabour
}

\section{Manal Mamadouh Youssef,}

\section{Maître de conférences- université de Minia- faculté d'Al-Alsun}

ABSTRACT Father of the negritude movement, poet and playwright, Aimé Césaire (1913-2008) was a descendant of former slaves deported from their native Africa to America. His poems and his plays defend the rights of negroes. His first theatrical work is "And the Dogs Silent" tells the story of a slave named the Rebel. He murdered his master and he faces death.

Salah Abdel Sabour is an Egyptian poet, editor, playwright and essayist (1931 -1981). He was a committed poet. He adopts a style marked by a meditative and pessimistic tone that will characterize his works. In his play "The Tragedy of Al-Hallaj" he recounts the tragedy of Mansur al-Hallaj who was a Persian mystic and lived in Baghdad.

Our investigation is interested in making a comparative study between the two heroes of these two pieces. Our problematic revolves around these questions: what are the elements of resemblance and dissimilarity between the two heroes? What are the facts that created the agreement or the disagreement? What are the common themes concerning the two heroes?

In our study, we will try to analyze the two plays from the perspective of Anne Ubersfled and the actantial model of Greimas.

\section{Keywords}

The hero, tragic, the theater, the actors, comparative literature 


\section{introduction}

La littérature comparée est parue au milieu du XIXe siècle. Le domine de la littérature comparée s'intéresse à détecter les éléments d'accord et de désaccord : "La littérature comparée est l'art méthodique, par la recherche de liens d'analogie, de parenté et d'influence, de rapprocher la littérature d'autres domaines de l'expression ou de la connaissance" (Pichois \& Rousseau, 1967, p. 174). La littérature comparée est donc l'art de comparaison. Si chaque comparaison exige trois éléments, le comparé, le comparant et l'outil de comparaison, la littérature comparée manque l'outil de comparaison. Le but final de la littérature comparée est de : "se tenir «au-dessus » des frontières et d'aspirer à être une étude, une science du «transnational »" (Pageaux, 1994, p. 18 ). C'est la discipline des frontières, le chercheur peut être à la fois dedans et dehors et peut aussi se situer aux carrefours de littérature.

Dans notre travail, nous traitons deux pièces, l'une est française et l'autre est arabe. Les deux pièces sont tragiques, c'est la figure du héros qui attire notre attention. Le héros est une figure essentielle dans le théâtre tragique et classique. Le premier dramaturge qui a mis en scène le premier héros tragique était Sophocle. En général, le héros tragique est confronté à des faits d'exception qui concerne la politique.

Aimé Césaire (1913- 2008) est l'un des écrivains noirs de langue française. Il est poète et dramaturge. Il défendait dans ses œuvres les droits des nègres. "Et les chiens se taisaient" (1958) est la première pièce de Césaire, elle se compose de trois scènes. Cette pièce est de pur produit de l'imagination, mais cela ne veut pas dire qu'elle n'a aucun ancrage dans la réalité; Césaire y exprime les choses qui lui tiennent à cœur à travers ses expériences réelles. La pièce raconte la vie d'un révolutionnaire, c'est un esclave qui, à cause de la maltraitance et de l'humiliation, a tué son maître et incité les nègres à la révolte contre la 
colonisation. Le héros doit affronter la mort, mais les autorités lui offrent d'exprimer ses remords et ses regrets en échange de sa vie. La pièce est une conquête de soi et de liberté où le héros a choisi la mort plutôt qu'une vie dans l'esclavage.

Salah Abdel Sabour (1931- 1981) est un poète, éditeur et dramaturge Égyptien. Il a étudié la littérature arabe, il enseignait la langue arabe et collaborait à la revue de Rose-al- Yusuf. "Massât-alHallaj" ou "La tragédie d'al-Hallaj" (1965) est son œuvre théâtrale la plus célèbre. " La tragédie d'al-Hallaj" se compose de deux parties, elle est inspirée d'un fait réel, c'est l'histoire de mort d'al-Husayn Mansur Al-Hallaj qui est un mystique et poète persan du soufisme sunnite. Al-Hallaj est condamné à mort et crucifié à Bagdad pour avoir parlé à des questions (politiques et religieuses) tabous.

L' investigation de la présente étude s'intéresse à faire une comparaison entre les deux héros de ces deux pièces. Notre problématique s'articule autour de ces questions : quels sont les éléments de ressemblance et de dissemblance entre les deux héros ? Quels sont les faits qui ont créé l'accord ou le désaccord ? Quels sont les thèmes communs concernant les deux héros?

Dans cette étude, nous essayerons d'analyser les deux pièces du théâtre sous la perspective d'Anne Ubersfled et le schéma actantiel de Greimas.

La présente étude se compose de quatre points : le premier point abordera le nom et l'appellation de chaque héros. Dans le deuxième point nous appliquerons le schéma actantiel de Greimas pour analyser les actants de chaque pièce. Le troisième point se focalise sur l'acteur en traitant les thèmes concernant le faire du héros. Le quatrième point abordera le rôle de chaque héros en montrant le rapport entre l'auteur et le héros. 


\section{Manal Mamadouh Youssef,. (BIJHS) Vol.3 Issue 1 (2021)}

Le héros est le personnage principal dans la pièce, le mot personnage "vient du latin persona, qui signifie le masque, c'est-à-dire le rôle tenu par l'acteur" (PARUNER, 2002, p. p.18 ). Le héros dans le théâtre est en général fictif ou historique tout en représentant une vision proposée au lecteur ou au spectateur.

Le personnage théâtral -surtout le héros- est celui qui unifie les différents signes de la pièce, c'est le personnage autour duquel s'articulent les thèmes de la pièce, c'est le personnage qui fait l'action de la pièce. Anne Ubersfeld voit que : "dans la mesure où le texte théâtral est essentiellement non linéaire mais tabulaire, le personnage est un élément décisif de la verticalité du texte ; il est ce qui permet d'unifier la dispersion des signes simultanés. " (Ubersfeld, 1996, p. 92).

Greimas redéfinit le concept du personnage dans la perspective structurale comme: une complexité qui contient trois niveaux : au niveau de la structure profonde, il est actant et au niveau de la structure de surface, il est acteur et rôle, au niveau de la structure superficielle, il est personnage (Greimas, 1966, p. 45).

Il s'avère important de montrer, ici, la perspective d'Anne Ubersfled, vue qu'elle redéfinit les notions suivantes : actant, acteur et rôle. Pour elle "un actant s'identifie à un élément, lexicalisé ou non, qui assume dans la phrase de base du récit une fonction syntaxique : il y a le sujet et l'objet, le destinataire, l'opposant et l'adjuvant, dont les fonctions syntaxiques sont évidentes ; le destinateur dont le rôle grammatical est moins visible, appartient si l'on peut dire à une autre phrase antérieure ou selon le vocabulaire de la grammaire traditionnelle, à un complément de cause" (Ubersfeld, 1996, p. 50)

L'acteur, d'après Anne Ubersfeld est : "la particularisation d'un actant ; il serait l'unité (anthropomorphe) qui manifesterait dans le récit la notion (ou la force) que recouvre le terme d'actant [...]. 
L'actant est un élément d'une structure syntaxique qui peut être commune à plusieurs textes; l'acteur est en principe l'acteur d'un récit ou d'un texte déterminé. Le même acteur peut passer d'une case actantielle à l'autre ou en occuper plusieurs" (Ubersfeld, 1996, p. 80). Tandis que le rôle est : "l'une des médiations qui permettent de passer $d u$ code actantiel abstrait aux déterminations concrètes du texte" (Ubersfeld, 1996, p. 84). Avant d'analyser l'actant, l'acteur et le rôle de chaque héros, nous tâcherons d'aborder, tout d'abord, leurs noms et leurs appellations dans chaque pièce pour connaître est-ce que le nom et l'appellation de chaque héros portent des significations symboliques? Y a-t-il une ressemblance entre ces significations ?

\section{I- Le nom et l'appellation}

Le nom, selon Roland Barthes, est "un instrument d'échange : il permet de substituer une unité nominale à une collection de traits en posant un rapport d'équivalence entre le signe et la somme." (Barthes, 1975 , p. 102). Le nom est un signe important qui donne plusieurs significations au personnage. Le nom débarrasse le dramaturge des détails volumineux sur l'identification individuelle et sociale.

Dans "Et les chiens se taisaient", Césaire donne à son héros un nom commun, c'est "Le Rebelle". C'est un nom et un adjectif à la fois. "Le Rebelle" dans le dictionnaire est celui : " qui refuse de se soumettre à une autorité, se révolte contre elle." (Amiel, 1987, p. 1332) la dénotation et la connotation du nom du héros apportent la même signification. Le héros dans la pièce est une figure qui lutte contre l'esclavage et l'occupation. Quand il se présente, il donne au lecteur une carte d'identité, il accentue la signification de son nom à travers son état:

\section{"LE REBELLE}




Je ne me soumets pas.

Mon nom : offensé ; mon prénom : humilié ; mon état: révolté; mon âge: l'âge de la pierre" (Césaire, 2000, p. 35)

Cette carte d'identité commence par "je ne me soumets pas", il refuse l'autorité qui stigmatise son nom et son prénom, c'est son état qui est le réacteur, il est "révolté", il se révolte contre l'oppression et l'humiliation.

Dans "La tragédie d'al-Hallaj", le héros est un personnage référentiel, le dramaturge n'a choisi ni le nom ni le prénom. Le héros s'appelle Al-Husayn Mansur Al-Hallaj. Al-Hallaj* c'est un surnom qui désigne le métier de son père qui travaillait dans le milieu textile. Mais c'est le prénom qui apporte une valeur significative, Al-Husayn désigne le petit-fils du prophète Mohamed, d'ailleurs ce prénom désigne le martyre $^{\dagger}$. Ce prénom est choisi consciemment de la part de sa mère. Il était très étonnant pour nous de lire l'extrait suivant :

"Quand sa mère tomba enceinte de lui, elle fit vœu de l'offrir comme serviteur à des «fuqara»(religieux pauvres volontaires). Et elle le nomma Hussein, en souvenir du fils martyrisé de Fátima, la fille bénie du prophète." (Ayouch, 2010, p. 134)

La mère qui a choisi le nom de son fils en souvenir Al-Husayn bin Ali, ne savait pas que son fils serait également un martyre: le martyre du soufisme.

Il convient de noter que le nom choisi par Césaire, dans la première pièce, et le nom référentiel, dans la deuxième pièce, tous les

* C'est un surnom qui signifie en français "cardeur de coton".

${ }^{\dagger}$ Husayn, Hussein, surnommé Sayyd ach-Chuhâdâ (prince des martyrs) (né le 8 janvier 626 mort le 10 octobre 680 lors de la bataille de Karbala) est le petit-fils du prophète Mahomed, fils d'Ali et de Fatima et le troisième des douze imams du chiisme duodécimain, à la mort de son frère aîné Hasan en 670. Sa mort fut un des éléments motivant les mouvements chiites. Réf : https://2u.pw/EPHyv 
deux partagent une valeur commune ; c'est le symbolisme. Le Rebelle symbolise la révolte et le prénom d'Al-Hallaj (Al-Husayn) symbolise le martyre.

À travers les appellations du héros, au lieu de son nom dans les didascalies, nous pouvons remarquer quelques points communs ou quelques points distincts. L'appellation peut également connoter quelques signes socio-politiques.

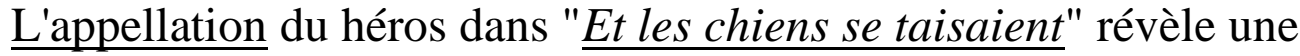
opposition où elle est variée entre la surévaluation et la sousévaluation. Deux manières opposées représentent deux pôles. Les nègres voient que le Rebelle est leur roi : "Vive le roi! (Césaire, 2000, p. 25) ". Alors que l'autorité représentée dans l'administrateur l'appelle différemment ; on l'appelle par des insultes, nous en dégageons deux exemples: "Chien, Gredin" (Césaire, 2000, p. 22)et "assassin, maudit." (Césaire, 2000, p. 51)

Dans "La tragédie d'Al-Hallaj", nous trouvons la même opposition entre deux pôles. Le peuple (surtout les pauvres) et les disciples d'Al-Hallaj l'appellent : "Mon seigneur" (Abdel-sabour, 1996, p. 32) et "Notre bon Cheick" (Abdel-sabour, 1996, p. 47). Alors que les juges et la police l'appellent d'une manière différente ; ils l'appellent "hérétique et infidèle" (Abdel-sabour, 1996, p. 92).

Cette opposition dans l'appellation désigne un conflit entre deux pôles, le héros appartient à l'un des deux. L'appellation désigne donc plusieurs oppositions. Dans "Et les chiens se taisaient", elle désigne l'opposition entre deux forces, le Rebelle appartient aux colonisés qui affrontent le colonisateur. Dans "La tragédie de 'Al-Hallaj", Al-Hallaj appartient au peuple et il affronte l'autorité (représentée dans la dynastie des Umayyades). L'opposition des classes sociales ; dans " $\underline{E t}$ les chiens se taisaient", le Rebelle appartient aux pauvres alors que le colonisateur possède toute la richesse du pays. Dans "La tragédie d'Al- 
Hallaj", al Hallaj appartient aux "Furka' (religieux pauvres), alors que les juges appartiennent à l'élite. Et dernièrement l'opposition morale, dans "Et les chiens se taisaient", le Rebelle défend un droit contre une autorité qui abuse de ce droit. Il défend la libération et l'indépendance. Dans "La tragédie d'Al-Hallaj", Al-Hallaj défend les droits des pauvres dont les pratiques de la dynastie, celle des Umayyades abusent. Ainsi est l'appellation un élément qui jette la lumière sur plusieurs autres questions. Pour bien aborder ces questions, nous traiterons l'actant, l'acteur et le rôle de chaque héros.

\section{II-L 'actant}

Le modèle actantiel de Greimas est une approche immédiate de la structure profonde de l'action. Le modèle actantiel se compose de six actants et se concentre sur trois axes. L'axe de transmission comprend le destinateur et le destinataire. L'axe de vouloir comprend le sujet et l'objet. L'axe de pouvoir comprend l'adjuvant et l'opposant. Le sujet est poussé par un destinateur qui l'incite à réaliser un désir (l'objet). L'adjuvant aide le sujet à réaliser son désir, alors que l'opposant contrarie le désir du sujet. Il s'agit en fin d'un destinataire qui bénéficie de la réalisation de la mission.

La figure suivante montre le modèle actantiel appliquée à

Le destinateur

(L'esclavage et la colonisation)

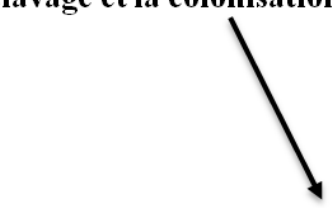

Le sujet (le Rebelle)

L'adjuvant (-) le destinataire

(le peuple)

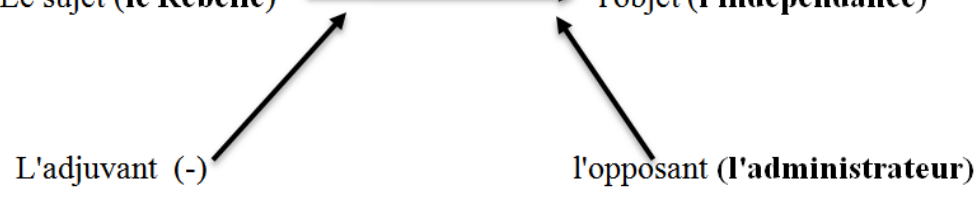


l'action dans "Et les chiens se taisaient":

Le destinateur qui incite le sujet à réagir est l'esclavage et la colonisation. La pièce désigne les deux questions : "le temps est plus complexe : il oscille entre le présent de la colonisation et le passé de l'esclavage" (Kesteloot \& Kotchy, 1973, p. 145). Le sujet c'est le héros, le Rebelle qui veut réaliser la libération et l'indépendance. Il est, comme le décrit l'administrateur, "le meneur" (Césaire, 2000, p. 8)qui incite le peuple à la révolte et qui a tué le maître : " Je l'ai tué de mes propres mains...Oui : de mort féconde... " (Césaire, 2000, p. 47). Le Rebelle est envahi par les souvenirs de l'esclavage et par la colonisation actuelle. Le ressentiment provoque en lui la haine, cette haine est le ferment de la liberté. Le Rebelle assassine le maître qui nie l'homme noir, l'assassinat émancipera le Rebelle de la longue servitude et affirmera l'irrésistible élan révolutionnaire en lui. L'oxymore dans "la mort féconde" désigne l'humiliation subie par le Rebelle qui veut débarrasser de ce maître. Le maître blanc incarne pour le Rebelle l'esclavage et la colonisation. Le Rebelle veut donner à son peuple l'exemple pour renoncer à sa peur. Le Rebelle doit choisir : regretter pour avoir tué le maître ou bien mourir. Le Rebelle se montre imperturbable et décide de mourir dans la dignité : "Et bien, je périrai" (Césaire, 2000, p. 113). Il est soumis à des tentations mais les menaces des geôliers ne prévaudront pas contre lui. Lylian Kesteloot a commenté cette instance en écrivant : "La mort du Rebelle c'est le prélude de sa renaissance et de celle de son peuple. Le héros disparu est plus puissant que jamais, car en Afrique l'esprit des ancêtres héros continue de régir les vivants" (Kesteloot \& Kotchy, 1973, p. 152).

L'objet c'est surement l'indépendance que le Rebelle voit comme le soleil dans une métaphore filée tout au long de la pièce : "Tu as tué le soleil, il n'y a plus de soleil" (Césaire, 2000, p. 51). La 


\section{Manal Mamadouh Youssef,. (BIJHS) Vol.3 Issue 1 (2021)}

personnification du soleil, qui désigne l'indépendance, montre que c'est une chose très précieuse et unique comme le soleil qui éclaire tout le monde. L'extrait suivant accentue sa demande :

\section{"L'ADMINISTRATEUR}

Je voulais savoir quels sont vos desseins.

\section{LE REBELLE}

C'est simple : vous chasser de ce pays... Le

rendre à ses légitimes propriétaires" (Césaire, 2000, pp. 105,106)

L'adjuvant dans "Et les chiens se taisaient", personne n'aide le Rebelle. Après les tentations de l'autorité, le peuple le laisse mourir. Sa mère, par l'amour maternel, s'adresse à sa sympathie pour le faire renoncer à son but :

\section{"LA MERE}

J'avais rêvé d'un fils pour fermer les yeux de sa mère.

\section{LE REBELLE}

J'ai choisi d'ouvrir sur un autre soleil les yeux de mon fils" (Césaire, 2000, p. 77)

Tandis que l'amante, par son amour voluptueux, s'adresse à l'instinct de survie :

"L'AMANTE

Embrasse-moi : la vie est là, le bananier hors des haillons lustre son sexe violet ; une poussière étincelle, c'est la fourrure du soleil, un clapotis de feuilles rouges, c'est la crinière de la forêt... 
Voici la Mort. Ses dents sont douces, là où fleurit la faim" (Césaire, 2000, p. 10)

Devant les deux tentations, le Rebelle ne renonce ni à son désir ni à la mission qu'il veut réaliser. Il préfère la mort.

L'opposant est représenté par le colonisateur. C'est l'administrateur avec sa force qui refuse l'indépendance. Il veut tuer le Rebelle pas par ses mains, mais par les mains du peuple : " le Rebelle ne mourra pas de nos mains. Je ne saurais que faire d'un martyr" (Césaire, 2000, p. 10). L'extrait suivant montre l'arrogance de l'administrateur devant la demande du Rebelle :

\section{"L'ADMINISTRATEUR}

Esclavage pour esclavage, attention!

Notre joug vaut mieux que celui des autres.

Vous ne serez jamais libres! Vous ne ferez que

changer de maîtres! " (Césaire, 2000, p. 110)

Le destinataire est le peuple qui bénéficiera de l'indépendance. Mais le Rebelle a peur d'être oublié par son peuple. Comme il est frappé par une vision prophétique, il prend peur de sa solitude, investi par le pressentiment qu'il sera renié par cette foule pour qui il se dévoue : "Assez, j'ai peur, je suis seul, mes forêts sont sans oreilles, mes pleurs sans chairs" (Césaire, 2000, p. 93). 
Dans la figure suivante, nous présentons la structure de l'action

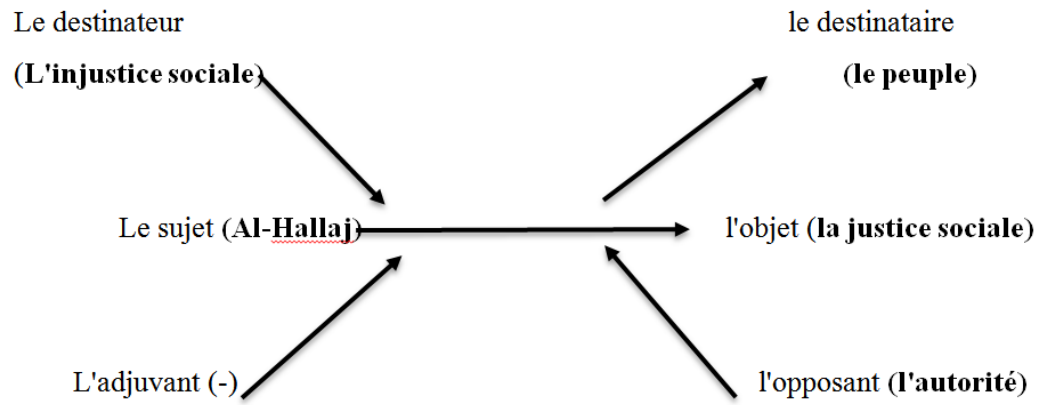

dans "La tragédie d'Al-Hallaj":

Le destinateur qui pousse le sujet à agir est l'injustice sociale, il s'agit d'une question qui inquiète le sujet:

"Al-Hallaj: qu'est-ce qu'on fait face au mal?

al-Shebly: le mal! Que veux-tu dire?

Al-Hallaj: la pauvreté des pauvres, la faim des affamés $\{. .$.

des hommes et des femmes qui ont perdu leur liberté

dis- moi, comment fermer les yeux sur tout cela?" (Abdel-sabour, 1996, p. 23)

Le sujet: c'est le héros, Al-Hallaj qui agit pour réaliser la justice sociale. Il voit que la pauvreté est le mal principal qui entraine tous les maux:

"Al-Hallaj: c'est quoi la pauvreté?

La pauvreté c'est l'oppression 


\section{La pauvreté c'est d'utiliser la pauvreté pour humilier l'âme}

La pauvreté c'est d'utiliser la pauvreté pour tuer l'amour et pour semer la haine" (Abdel-sabour, 1996, p. 112)

Il critique l'injustice sociale qui ronge la cohésion de la société :

"La pauvreté dit aux fortunés : détestez les pauvres ! Ils souhaitent la disparition de vos fortunes.

La pauvreté dit aux pauvres : si vous avez faim, mangez la chair de vos frères !" (Abdel-sabour, 1996, p. 112)

Al-Hallaj a envoyé des messages à Al-Madhrai et Hamad AlTouluni et Al-Qen (qui sont connus par leur opposition contre l'autorité des Umayyades) pour leur demander de trouver une solution de l'injustice sociale. Mais Al-Hallaj est, en premier lieu, le mystique qui apparaît dans les souks pour prêcher sa doctrine mystique*. Al -Hallaj qui s'est plongé dans la méditation d'Allah, se trouve non seulement à un "ittisâl" (contact entre l'âme de l'homme et Allah) mais à un "hula" (inhabitation) où l'esprit d'Allah habite, sans confusion de nature, à l'âme purifiée du mystique (Al-Hallaj) ${ }^{\S}$. Quand Al-Hallaj commence à parler de de "hula" l'autorité voit que c'est une bonne occasion pour arrêter Al-Hallaj. Pour l'autorité, les accusations contre lui ne manquent pas :

\$ La Science des cœurs est posée par le texte du Coran qui en pose les bases, science approfondie par les soufis. C'est par son cœur que l'homme entre dans la contemplation du divin. L'homme a différentes enveloppes successives qu'il lui faut traverser et qui correspondent au degré de purification de l'âme. Pour Hallaj, trois phases dans la voie, ou l'ascension : phase de pénitence, ou ascèse des sens ; phase de purification massive (on se détache de son moi subjectif) ou s'instaure une réciprocité de l'amour et du désir entre Dieu et sa créature; et enfin phase de l'ascèse de l'esprit qui débouche sur l'union avec le divin. Le soufi a alors conquis le droit de dire le « je » qui l'unit à la source même de la parole divine car il atteint alors le stade de l'union transformante avec Dieu. (Ayouch, 2010, p. 138)

$\S$ Selon la vision d'al-Hallaj. 
" Dénoncé comme agitateur politique, organisateur de réunions secrètes. De plus, son exaltation, les sacrifices qu'il s'inflige, exacerbent la vindicte des autorités religieuses. Mais ont pu s'entremêler des raisons sociales et politiques, des conflits d'intérêts entre des partis, des antagonismes entre les différents appuis et les équilibres" (Ayouch, 2010, p. 142)

L'autorité lui envoie des policiers déguisés en hommes pauvres, ils écoutent ses paroles et quand il arrive à "hula", alors ils annoncent et crient que "c'est bien la varie hérésie" (Abdel-sabour, 1996, p. 48). Al Hallaj a été arrêté et mis en prison.

L'objet est la justice sociale qu'Al-Hallaj veut réaliser. AbdelSabour paraît habile en donnant un des résultats de l'injustice sociale pour pointer clairement l'influence subversive de l'injustice sociale. C'est l'histoire d'un prisonnier, qui était en prison avec Al-Hallaj, cette histoire montre que ce prisonnier était un enfant passionné pour les sciences :" tu sais, mon bon cheik, un jour quand j'étais enfant et innocent, j'aimais les mots" (Abdel-sabour, 1996, p. 74), les mots ici sont une périphrase des sciences. C'est la mère de ce prisonnier qui l'encourage à apprendre les sciences, sa mère était une servante, elle est morte à cause de la pauvreté : " ma mère n'est pas morte à cause de la faim, ma mère est habituée à la faim, donc, elle est tombée malade au matin, elle a vieilli après-midi et elle est morte avant le soir" (Abdelsabour, 1996, p. 75). Cette petite histoire désigne comment l'injustice sociale peut entraîner la déviation : l'enfant qui était passionné pour la science est devenu un criminel.

L'adjuvant : personne n'aide Al-Hallaj à réaliser son désir. Ses disciples laissent Al-Hallaj mourir devant les yeux : "Le groupe des soufistes : nous aimons ses mots plus que lui, donc, nous le laissons mourir afin que ses mots vivent" (Abdel-sabour, 1996, p. 11) Al-Shably lui demande de ne pas parler de "hula" car c'est une question privée 
entre l'homme et Allah. Al-Madhrai et Hamad Al-Touluni et Al-Qeni s'enfuient.

L'opposant : l'opposant dans "La tragédie d'Al-Hallaj" est l'autorité représentée par les juges. Après un long procès, l'autorité a envoyé un message aux juges indiquant qu'elle a pardonné Al-Hallaj pour l'agitation politique mais elle ne pourrait pas lui pardonner pour l'infidélité. Au procès, il y a trois juges : Ibn Surayj, Abu Omar alHammadi et Ibn Suleiman, les deux derniers sont toujours contre AlHallaj, ils ne lui donnent pas la chance pour parler, ils ont un seul but : prononcer la peine de mort contre Al-Hallaj pour rendre l'autorité satisfaite. Alors que Ibn Surauj voit que la question de "hula" est une question entre le mystique et Allah, on ne doit pas le punir pour cette question. Il quitte la séance à la fin de la pièce : "mais c'est une question entre l'homme et son créateur, notre tribunal ne statue pas sur ces questions" (Abdel-sabour, 1996, p. 107).

Le destinateur est le peuple, mais son rôle est très passif.

\section{III- L'acteur}

À ne pas confondre avec le comédien, l'acteur se caractérise par ce que le héros a à faire. Dans ce point, nous exposons "le faire" du héros. En étudiant les deux pièces, nous trouvons que les deux héros ont

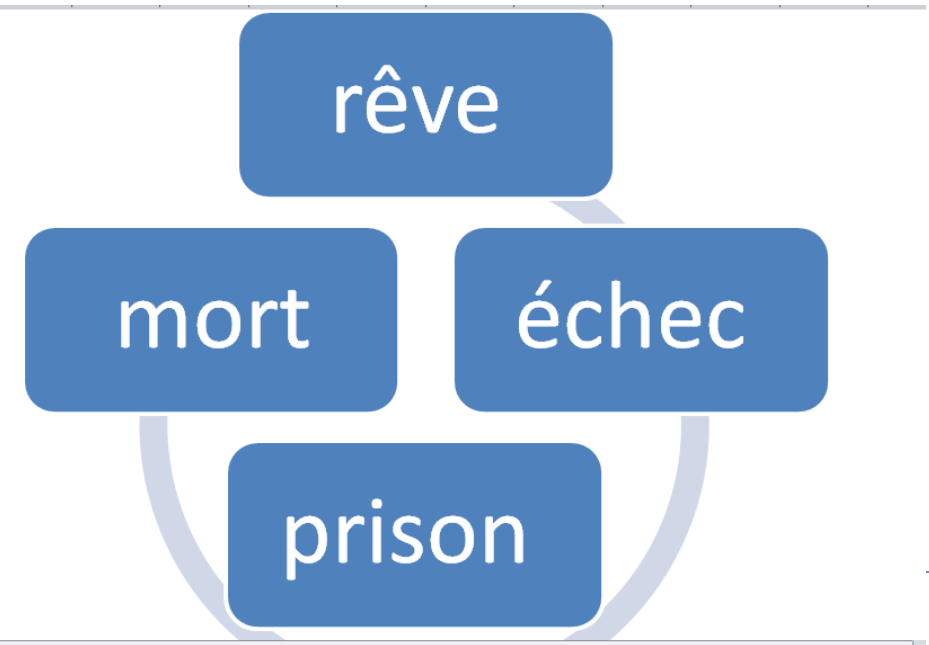




\section{Manal Mamadouh Youssef,. (BIJHS) Vol.3 Issue 1 (2021)}

traversé un cercle commençant par un rêve et se terminant par la mort:

\section{Référence}

\section{Le rêve :}

Dans "Et les chiens se taisaient", le Rebelle annonce clairement son rêve en disant : "Mes amis, j'ai rêvé de lumière, d'enseignes d'or, de sommeils pourprés de réveils d'étincelles et de peaux de lynx" (Césaire, 2000, p. 11). L'arme du Rebelle dans sa bataille est les mots : "Il combat avec les mots (Césaire, 2000, p. 6)". Il utilise les mots pour haranguer la foule :

\section{" LE REBELLE}

(qui tient la foule en haleine)

Et vous n'avez plus la force de protester, de

vous indigner, de gémir,

condamnés à vivre en tête à tête avec la stupidité empuantie, sans autre chose qui vous tienne chaud au sang que de regarder ciller" (Césaire, 2000, p. 6)

Dans "La tragédie d'al Hallaj", c'est la pauvreté qui incite AlHallaj à réagir. Le rêve d'Al-Hallaj est de réveiller le peuple, son arme est celle du Rebelle : les mots. Abdel Sabour a consacré la première partie de sa pièce qui est intitulé "Le mot" pour montrer l'influence des mots d'Al-Hallaj sur ses disciples et sur le peuple. Il emploie les mots pour inciter le peuple et pour avertir les dirigeants.

"Al-Hallaj: Je espère réveiller les morts. 
Le deuxième prisonnier : (ironiquement) est -tu un second Christ $? !$

Al-Hallaj: tu n'es pas compris mon fils. Pour revivifier un corps, tu dois posséder la position ou le miracle du Christ, mais pour réveiller un esprit, il suffit de posséder les mots" (Abdel-sabour, 1996, p. 70).

\section{L'échec}

Dans "Et les chiens se taisaient", sur le plan purement humain, nous pouvons dire que le Rebelle a échoué. Matériellement, le Rebelle sera mort, sa mère n'aura plus personne pour l'ensevelir. Gorges N'gal explique pourquoi le Rebelle a échoué : "c'est parce qu'il rêve l'impossible, le démesure et l'absolu" (Owusu-Sarpong, 2002, p. 115). Pour nous, l'échec du Rebelle peut s'expliquer par certaines causes : les forces oppressives, la complicité, l'incompréhension et la maladresse.

Les forces oppressives : l'arrestation du Rebelle, l'exercice des forces coercitives sur sa personne et sa mort en fin montrent dans quelle mesure l'oppresseur le domine par la force militaire.

La complicité : Césaire désigne une cause très importante de l'échec du Rebelle, c'est la complicité de certains nègres comme le Vendu, qui est un nègre servante chez le colonisateur. Le Vendu (comme son nom l'indique) vend tout pour l'argent. Il frappe et humilie le Rebelle pour obtenir l'argent :

" Fais ce que j'ai dit. Va-t'en! Je te récompenserai de pièces de cuivre.

(II jette quelques pièces de monnaie au Vendu qui s'empresse de les ramasser" (Césaire, 2000, p. 8)

L'incompréhension : le but du Rebelle n'est compris ni de sa mère ni de son amante ni de son peuple pour lequel il est en train de se 


\section{Manal Mamadouh Youssef,. (BIJHS) Vol.3 Issue 1 (2021)}

sacrifier. C'est vraiment le peuple qui devait réagir. Si le peuple réagit, il renverser complétement la situation. Il peut faire deux coups d'une seule pierre : sauver le Rebelle et obtenir sa liberté.

La maladresse : l'acte du Rebelle est certainement indispensable, mais il est cependant maladroit. Il n'avait pas pris assez de précaution pour mettre le peuple au courant de ses objectifs. Le peuple ne réagit parce qu'il ne lui pas possible d'agir conformément au plan du Rebelle.

Dans "La tragédie d'Al-Hallaj", nous trouvons que ce sont les mêmes causes qui entrainent l'échec d'Al-Hallaj. L'autorité a exploité ses forces pour horrifier Al-Hallaj. Ibrahim, l'un des disciples d'AlHallaj, avertit Al-Hallaj du risque et lui conseille de s'enfuir: " Les gouverneurs vous ont réservé des trucs" (Abdel-sabour, 1996, p. 27).

La complicité est parue clairement dans la réaction du grand public. Le grand public, au lieu de défendre Al-Hallaj, ils ont participé à sa mort. Ils vendent l'affaire pour obtenir l'argent. Abdl-Sabour désigne cette scène par des mots très expressifs :

" Ils nous ont classés, l'un après l'autre

le plus bruyant et le plus long

sont classés au premier rang

le moins bruyant et le relâché

sont classés au deuxième rang

ils ont donné à chacun un dinar

un dinar d'or brillant

Personne ne l'a déjà touché 
ils ont dit: "criez qu'il est hérétique et infidèle"

nous avons cri: " hérétique et infidèle!"

ils ont dit:" criez qu'l'on le tue, nous aurons du sang sur nos mains"

nous avons cri : "Que l'on le tue, nous aurons du sang sur nos mains". (Abdel-sabour, 1996, p. 10)

Al- Hallaj a également commis la même faute du Rebelle, il s'est sacrifié pour son peuple. Mais il n'a ni éduqué ni informé son peuple, c'est pourquoi le peuple n'a pas répondu à ses attentes. Son ami AlShebly l'a informé que le peuple n'assumera pas la responsabilité : " AlShebly: "Ne t'ai-je pas mis en garde contre le grand public? Est-ce que ce monde mérite de se sacrifier?" (Abdel-sabour, 1996, p. 16)

\section{La prison}

Chaque héros dans chaque pièce représente une valeur, il lutte tout en refusant les essais qui opposent cette valeur. Dans "Et les chiens se taisaient", le Rebelle représente la libération du Nègre colonisé. Il ne peut pas exister en dehors de cette valeur qui forme son idéal. Le Rebelle refuse la famille représentée par la mère, l'amour représenté par l'amante et la force représentée par le colonisateur. Dans la prison les deux héros ont été humiliés et battus :

\section{" LEGEOLIER}

Crois-tu que nos coups lui fassent mal ? en tout cas ça ne marque pas.

(Il frappe.)

Oh, oh, son sang coule. 


\section{LE MESSAGER}

Frappe-le, frappe-le, cela fera du bien à sa vilaine couenne" (Césaire, 2000, p. 116)

Dans "La tragédie d'Al-Hallaj", Al-Hallaj a été aussi battu et insulté en prison. Mais nous constatons que le Rebelle utilise la violence verbale : " Cuve de scorpions" (Césaire, 2000, p. 12)et, " Malheur sur vos têtes ! " (Césaire, 2000, p. 13), alors qu'Al-Hallaj, qui est un religieux, utilise des paroles aimables : " ne m'insulte pas mon fils, l'insulte est un péché". (Abdel-sabour, 1996, p. 66)

\section{La mort}

Les deux héros ont été emprisonnés, et ont été tués par l'autorité dirigeante. Les deux héros ont affronté la mort avec héroïsme. Les deux héros ont surmonté la peur et la menace. Les deux héros sont restés lucides dans la révolte et dans le refus. Mais la mort de deux héros n'est qu'un début pour le peuple qui devait réagir mais il reste faible et n'ose pas de dire non : " Le Rebelle meurt, l'espoir au cour. Sa mort sera pour les siens le début de la fécondation" (Owusu-Sarpong, 2002, p. 118). Les deux héros se sacrifient en total pour leurs siens, pour leurs peuples pour tous les opprimés.

\section{IV-Le rôle du héros: rapport entre le héros et le dramaturge}

L'étymologie du terme (rôle) renvoie au rouleau de bois sur lequel était fixé le texte du comédien (PARUNER, 2002, p. 80). Le rôle désigne la fonction particulière qui incombe à l'auteur : "Elle permet "de passer du code actantiel abstrait aux déterminations concrètes du texte" (PARUNER, 2002, p. 80). Pour montrer le rôle de chaque héros, nous devons passer du texte au temps de l'écriture pour s'interroger sur les raisons qui poussent les deux dramaturges à écrire leur texte. Dans "Et les chiens se taisaient", le Rebelle représente le nègre révolté dont 
le pays est colonisé. Le Rebelle demande l'émancipation et l'indépendance. Dans "La tragédie d'Al-Hallaj", Al-Hallaj représente le religieux pauvre qui demande la réforme politique et sociale.

Il s'avère important de s'interroger sur le temps de l'écriture, pourquoi chaque dramaturge a choisi ce type du héros à ce temps-là ? Césaire a écrit sa pièce en 1958, Abdel-Sabour a écrit sa pièce en 1964, alors, les deux pièces sont écrites, presque, à la même époque.

Pour "Et les chiens se taisaient", il faut noter qu'avant la Deuxième Guerre mondiale il était impossible pour les nègres de penser à l'indépendance : " les termes "émancipation" et "indépendance" étaient ignorés sinon par tous, du moins par la majorité de la population en Afrique noire" (Mbom, 1979, p. 33). Mais quand la guerre arrive, les maîtres font appel à leurs esclaves pour lutter à leurs côtés. Cette situation a bouleversé les rapports entre les nègres et les maîtres : ils parlaient et mangeaient ensemble, ils, même, partageaient la même couche : "Au combat, on était légal de son maître" (Mbom, 1979, p. 33). Les nègres ont gagné des promesses pour l'indépendance, mais après la guerre, on a découvert que ces promesses étaient fallacieuses. Césaire voit que c'est le temps pour inciter les nègres à demander l'émancipation et l'indépendance, c'est pourquoi, il a écrit "Et les chiens se taisaient".

Pour " La tragédie d'Al-Hallaj", Abdel- Sabour a choisi un personnage référentiel pour évoquer le problème, de son époque, qui était entre le poète et l'autorité : " Par le procès d'Al-Hallaj, AbdelSabour veut exposer la question du poète et sa lutter contre l'autorité de cette époque- en 1964-" (Ismail, 2017, p. 212) Abdel-Sabour voulait mettre en relief l'importance de la liberté d'expression, pour cette raison, nous trouvons que l'arme d'Al-Hallaj est les mots. Pour l'histoire réelle, l'accusation d'Al-Hallaj était religieuse, mais, dans la pièce, la question religieuse devient secondaire alors que la question politico- 


\section{Manal Mamadouh Youssef,. (BIJHS) Vol.3 Issue 1 (2021)}

sociale devient la question principale. Dans la pièce, pendant le procès d'Al-Hallaj, nous comptons quatorze pages pour interroger Al-Hallaj sur la question de la réforme qu'il demande. Pour la question religieuse, Abdel-Sabour se contente de l'inciter en quelques mots :

" Ibn Sarij (le juge) : est-ce que tu crois en Allah?

Al-Hallaj : Il est notre Créateur, c'est vers lui que nous retournerons". (Abdel-sabour, 1996, p. 116)

Après avoir exposé le temps de l'écriture, nous pouvons déduire que les deux héros dans les deux pièces représentent en fait le rôle de l'auteur dans la société.

\section{Conclusion}

Bien que le héros de "Et les chiens se taisaient" soit de pur produit de l'imagination de Césaire, nous trouvons une ressemblance étonnante avec le héros référentiel de "La tragédie d'Al-Hallaj" de Abdel-Sabour. L'analyse sémiotique désigne que les noms des deux héros partagent la même valeur : le symbolisme. Le nom de chaque héros symbolise une valeur morale : le Rebelle symbolise la révolte, Al-Hallaj symbolise le martyre. L'appellation de chaque héros montre l'opposition entre deux pôles, cette opposition désigne l'opposition politique et sociale dans chaque société.

L'étude du schéma actantiel, dans chaque pièce, a mis en relief les faits qui ont créé la ressemblance entre les deux héros. Les deux héros sont les sujets qui veulent réaliser un désir dont le profit est public. Bien que le dessinateur soit différent mais l'objet de chaque héros est 
un objectif noble et humain. L'opposant est le même pour chaque héros : l'autorité. Le destinataire est le même, c'est le peuple. Mais personne n'aide les deux héros.

Les deux héros ont traversé un cercle qui commence par un rêve et se termine par la mort en passant par l'échec et la prison. Même les causes qui entrainent l'échec sont les mêmes pour chaque héros.

Pour étudier le rôle de chaque héros, nous avons recouru au temps de l'écriture pour connaître les raisons qui ont incité les deux dramaturges à choisir le rôle du héros dans chaque pièce. Chaque dramaturge a évoqué son désir personnel à travers son héros. Césaire a voulu inciter les nègres colonisés à demander l'indépendance, AbdelSabour a évoqué le problème de son époque qui était entre lui et l'autorité, c'est la question de la liberté d'expression.

\section{Bibliographie}

\section{I- Corpus}

Abdel-sabour, S, (1996). La tragédie d'Al-Hallaj". Le Caire : librairie Al-Osra.

Césaire, A, (2000). "Et les chiens se taisaient". Paris : Editions Présence Africaine.

\section{II- ouvrages Généraux}

ALLEN, J, (2017)," Contraindre le chaos à devenir forme : le motif de la descente aux enfers dans Et les chiens se taisaient d'Aimé Césaire", Leeds, Journal of Postcolonial Writing, 54 (2-3). pp. 185-201. ISSN 1744-9855

Amiel, E, (1987). "Hachette : dictionnaire du français". Paris: Hachette.

Aron, P, Saint-Jacques, D., \& Viala, A. (2002). " Le Dictionnaire du Littéraire". Paris: PUF. 


\section{Manal Mamadouh Youssef,. (BIJHS) Vol.3 Issue 1 (2021)}

Ayouch, S, (2010). "La passion de husayn mansûr Al-Hallaj". L’Esprit du temps (4 n 113), p. pages 133 à 147.

Barthes, R, (1975). "S/Z", Hill and Wang.

De Saussure, F, (1915). "Cours de linguistique générale". Genève.

Greimas, A. J. (1966). "Sémantique Structurale", Paris : éd. Larousse.

HATEM, J, (2005) " Hallaj et le Christ", Paris, Harmattan

Ismail, A. (2017). "L'impact de l'héritage arabe sur le théâtre égyptien contemporain". Royaume-Uni : Fondation Hindawi,

Kesteloot, L., \& Kotchy, B. (1973). « Aimé Césaire, l’homme et l'œuvre ». Paris: Présence Africaine.

Mbom, C, (1979). "Le théâtre d'Aimé Césaire : ou la primauté de l'université humaine". Paris: Nattan.

Owusu-Sarpong. (2002). "Le temps historique dans l'œuvre théâtrale d'Aimé Césaire". Paris : (Albert), Harmattan

Pageaux, D.-H. (1994). "La littérature générale et comparée". Paris: Armand Colin.

Paruner, M. (2002). "L'analyse de texte de théâtre", Paris, Nattan, "Lettres sup" 2002, p. 69. Paris: Nattan.

Pichois, C, \& Rousseau, A.-M. (1967). "La littérature comparée". Paris : Armand Colin,

RUHE, E, (1990),"Ailné Césaire et Janheinz Jahan: les débuts du théâtre césarien", Würzburg, Konigshausen \& Neumann

Ubersfeld, A. (1996). "Lire le théâtre". Paris : Belin.

YAZID, M, (2014),"Analyse sémiologique des personnages dans RUE DARWIN", Béjaïa, Université Abderrahmane-mira Bejaia 